ACCEPTED MANUSCRIPT

\title{
Evaluating silver-plated nylon (Ag/PA66) e-textiles for bioelectrical impedance analysis (BIA) application
}

To cite this article before publication: Irini Logothetis et al 2020 Meas. Sci. Technol. in press https://doi.org/10.1088/1361-6501/ab78c3

\section{Manuscript version: Accepted Manuscript}

Accepted Manuscript is "the version of the article accepted for publication including all changes made as a result of the peer review process, and which may also include the addition to the article by IOP Publishing of a header, an article ID, a cover sheet and/or an 'Accepted

Manuscript' watermark, but excluding any other editing, typesetting or other changes made by IOP Publishing and/or its licensors"

This Accepted Manuscript is @ 2020 IOP Publishing Ltd.

During the embargo period (the 12 month period from the publication of the Version of Record of this article), the Accepted Manuscript is fully protected by copyright and cannot be reused or reposted elsewhere.

As the Version of Record of this article is going to be / has been published on a subscription basis, this Accepted Manuscript is available for reuse under a CC BY-NC-ND 3.0 licence after the 12 month embargo period.

After the embargo period, everyone is permitted to use copy and redistribute this article for non-commercial purposes only, provided that they adhere to all the terms of the licence https://creativecommons.org/licences/by-nc-nd/3.0

Although reasonable endeavours have been taken to obtain all necessary permissions from third parties to include their copyrighted content within this article, their full citation and copyright line may not be present in this Accepted Manuscript version. Before using any content from this article, please refer to the Version of Record on IOPscience once published for full citation and copyright details, as permissions will likely be required. All third party content is fully copyright protected, unless specifically stated otherwise in the figure caption in the Version of Record.

View the article online for updates and enhancements. 


\title{
Evaluating silver-plated nylon (Ag/PA66) e-textiles for bioelectrical impedance analysis (BIA) application
}

\author{
Irini Logothetis ${ }^{1,2}$, Derman Vatansever Bayramol ${ }^{3}$, Ignacio Gil ${ }^{2}$, Peter Dabnichki ${ }^{4}$, \\ Elena Pirogova ${ }^{1}$ \\ ${ }^{1}$ Discipline of Electrical and Biomedical Engineering, RMIT University, Melbourne, Australia \\ ${ }^{2}$ Department of Electronic Engineering, Universitat Politècnica de Catalunya, Terrassa (Barcelona), \\ Spain \\ ${ }^{3}$ Department of Textile Engineering, Namik Kemal University, Corlu-Tekirdag, Turkey \\ ${ }^{4}$ Discipline of Mechanical and Automotive Engineering, RMIT University, Melbourne, Australia
}

E-mail: irini.logothetis@ student.rmit.edu.au

Received $\mathrm{xxxxxx}$

Accepted for publication $\mathrm{xxxxxx}$

Published xxxxxx

\begin{abstract}
Bioelectrical Impedance Analysis (BIA) is an established method for assessing integrity of biotissue. Adapting BIA as a diagnostic tool to monitor electrophysiological activity gives rise to evidence-based objective diagnostic approaches as opposed to visual assessment currently performed by practitioners in wound healing management. Advancements in the telecommunication and textile industries have made the Internet of Wearable Things (IoWT), the future of telemedicine. E-textile electrodes give us the ability for long-term monitoring applications; however, they are associated with electrode polarization impedance $\left(Z_{p}\right)$ contributing to the electrode-skin impedance $\left(Z_{e s}\right)$. By studying the design of e-textile electrodes, we can reduce $Z_{p}$ and characterise it relative to changes in skin properties, such as skin temperature and perspiration. In this study, we examined the effects of selected textile substrates on changes in $Z_{p}$ of e-textile electrodes, and characterized $Z_{p}$ in a climatic chamber with temperature and relative humidity settings emulating skin temperature and perspiration. An air permeability test was also conducted to account for the physiological comfort of the etextile electrodes. Our results demonstrate that a polyester non-woven felt substrate is ideal for use in e-textile electrodes. By understanding and quantifying the relationship between $Z_{p}$, skin temperature and perspiration, this insight can be incorporated into the calibration process of BIA systems for accurate long-term monitoring resulting in an objective assessment of changes in tissue integrity.
\end{abstract}

Keywords: electronic textile (e-textile) electrodes, bioelectrical impedance analysis, polarization impedance, electrode test setup, wearable electrodes, biopotential electrodes

\section{Introduction}



Research into adapting Bioelectrical Impedance Analysis (BIA) to monitor electrophysiological activities as a diagnostic tool [1-14] offers opportunities to reduce costs and improve efficiency in the health system while improving the 
quality of life for patients. By adapting this technology to patients' monitoring in home settings, the length of hospital stays, number of hospital readmissions and visits to emergency departments can be reduced [15]. Nowadays the advancement of the internet has led to the birth of Internet of Things (IoT) advancing telemedicine. In conjunction to this technology, the emergence of conductive yarns has evolved the fabrication process of e-textiles, thus providing an opportunity for replacing current clinical electrodes and introducing a new era of Internet of Wearable Things (IoWT).

E-textile electrodes are the most convenient form of dry electrodes enabling long-term monitoring of patients' electrophysiological activity. Its simple fabrication process provides versatility which allows development of customised e-textile electrodes for medical applications specific to a patient's ailment [16-19]. Up-to-date, research into e-textile electrodes has been adapted for measuring: (i) ECG, (ii) EEG and EMG signals at low frequencies ranging between (i) 0.5 $\mathrm{Hz}$ to $100 \mathrm{~Hz}$, (ii) 0.5 to $42 \mathrm{~Hz}$ and (iii) 20 to $500 \mathrm{~Hz}$ [18-34]. However, e-textile electrodes are associated with electrode polarization, $Z_{p}$, resulting in signal attenuation and distortion. For a clear and concise biosignal reading, it is important for etextile electrodes to have a low $Z_{p}$ [35].

Our previous study [35] demonstrated the influence of embroidery characteristics on $Z_{p}$ for single frequency BIA (sfBIA), at the predefined frequency of $50 \mathrm{kHz}$. By adapting an electrode wafer experimental methodology to isolate $Z_{p}$, we showed that increasing the surface area of e-textile electrodes results in the reduction of $Z_{p}$, which was also influenced by the embroidery characteristics, such as stitch type, length and spacing (stitch density). However, e-textile electrodes come into direct contact with the human skin. Therefore, the design necessitates consideration of variances in skin conditions, such as skin temperature and perspiration relative to the skin and electrode contact area. This, in addition to $Z_{p}$, gives rise to an electrical impedance between the skin and electrode, namely the electrode-skin impedance $\left(Z_{e s}\right)$ [36-39]. By adapting BIA as a diagnostic tool connected to coupled etextile electrodes, $Z_{e s}$ contributes to the measured impedance $\left(Z_{m}\right)$ of the biotissue under study (BUS), where $Z_{m}$ by the system is represented below:

$$
Z_{m}=Z_{B U S}+Z_{e s}+N, \text { thus } Z_{B U S}=Z_{m}-Z_{e s}-N
$$

where $Z_{m}$ is the measured impedance by the BIA system, $Z_{B U S}$ is the impedance of the biotissue under study (BUS), $Z_{e s}$ is the electrode-skin impedance and $N$ refers to all other potential noise, such as motion and pressure artefacts [40-43]. For the purpose of this study, we are focusing on $Z_{p}$ of e-textile electrodes as a standalone parameter.

It is known that $Z_{e s}$ is an ongoing and major issue in measuring electrophysiological activity of biotissues. Clinical wet electrodes, $\mathrm{Ag} / \mathrm{AgCl}$, are manufactured with an electrolyte 'gel' layer forming an ideal non-polarized electrode [44] contrary to e-textile electrodes. Thus, they are distinguished for their low $Z_{e s}$. However, the main issue pertaining to wet electrodes is the dehydration of the 'gel' layer and adhesiveness resulting in: (i) varied $Z_{m}$, making it difficult to deduce $Z_{B U S}$; and (ii) a greater probability of $Z_{e s}$ mismatch, which can cause excessive powerline interference contributing to $N$ [45]. Wet electrodes are limited in enabling control of monitoring changes in skin properties over a longterm period. These issues, however, can be addressed by adopting e-textiles with low $Z_{p}$ and deducing the influence of skin properties, i.e. perspiration and temperature, for accurate and consistent measurements. Also, by selecting an appropriate substrate in the design process, we can fabricate an e-textile with a reduced $Z_{p}$ in conjunction with a relatively close to 'zero-slope' linear regression of $Z_{p}$ correlating to changes in skin properties. Evaluating the effects of perspiration and skin temperature on changes in $Z_{p}$ can assist in further understanding of $Z_{e s}$ that can assist in extracting $Z_{B U S}$ from $Z_{m}$

\subsection{Physiological influences from skin}

The temperature of the skin not only varies with the environmental temperature or with the body core temperature but also with conditions in the skin and its underlying structure. This is the reason for variances in skin temperature in different regions of the human body. Skin temperature is expected to be lower over (i) protruding parts, such as nose, ears, fingers and toes; (ii) bone or tendons; (iii) over veins; or (iv) organs at rest. It is higher over muscles, arteries and active organs [46]. In addition, medical conditions of a patient can cause a disruption to regular bodily structure and function which can increase or decrease the skin temperature. To defeat infectious agents such as viruses and bacteria, the body's immune response increases the body's temperature [47]. This increases the skin temperature and the body produces sweat to cool the body down in order to maintain its thermophysiological temperature. Moreover, surgical procedures can also decrease the body's temperature [48]

Of particular relevance to this study is the thermophysiological skin temperature. Interestingly, research into determining a threshold for the thermophysiological skin temperature is uncertain. Bierman in 1936 [46] suggested skin temperature varies between 33.5 and $36.9^{\circ} \mathrm{C}$. More recent studies indicate that the skin temperature can vary between $30^{\circ} \mathrm{C}-34^{\circ} \mathrm{C}$ [49-51]. A study by Mole et al. relates the relative humidity $(\mathrm{RH})$ to skin wetness correlating the wetness of the skin to an increase in $\mathrm{RH}$ over $40 \% \mathrm{RH}$ between the temperature range of $30^{\circ} \mathrm{C}-34^{\circ} \mathrm{C}$ [52]. Hence, these boundary conditions must be considered in characterizing $Z_{p}$ of e-textile electrodes relative to the influence of skin changes. In addition, the microclimate between the skin and the e-textile electrode can influence the skin temperature. By studying the 
breathability, an e-textile electrode with low air permeability increases the skin temperature resulting in perspiration [53] (Appendix I). Therefore, it is important to consider an e-textile electrode possessing the largest air permeability to assist in the thermoregulation of the skin.

In this study, we consider the physiological influences stemming from skin temperature and perspiration on etextiles. By adapting current knowledge of these parameters, we formed a methodological protocol to characterize $Z_{p}$ of various e-textile electrodes. Initial tests were conducted in an ambient environment before testing the electrodes in a climatic chamber, where the temperature and $\mathrm{RH}$ were used to emulate human skin temperature and perspiration. To evaluate the selected substrates and thus, identify the substrate suitable for BIA analysis, the air permeability was also tested across all fabricated e-textile electrodes.

\section{Methods}

The fabrication of efficient e-textile electrodes requires selection of appropriate materials, such as conductive yarn and substrate. However, skin temperature affects the temperature of the e-textile electrode, while perspiration results in an increase of the textile moisture content impacting $Z_{p}$. This affects the performance of the e-textile electrodes (Fig. 1b) resulting in unstable diagnostic measurements. Therefore, suitability of common commercial textile materials of various structures as substrates (S1: cotton plain weave, S2: cotton poplin, S3: cotton velvet and S4: polyester non-woven felt) for e-electrodes was evaluated. By tailoring these e-textile electrodes, we can develop a BIA-based diagnostic tool for long-term monitoring of the electrophysiological activity of a patient as shown in Fig. 1.

\subsection{E-textile electrode fabrication}

Table 1 shows the textile substrates with the pertinent characteristics including: (i) the composition of the textile, (ii) its structure, (iii) weight in grams per square meter $\left(\mathrm{g} / \mathrm{m}^{2}\right)$ and (iv) relative permittivity $\left(\varepsilon_{r}\right)$. The relative permittivity for the textile substrates was measured using a split post dielectric resonator (QWED, Warszawa, Poland), a brief explanation of relative permittivity can be found in Appendix I. These characteristics are a guideline to understanding the fundemental differences in the selected substrates relative to one another. To distinguish the structure of the substrates Scanning Electron Microscopy (SEM) was used, with the SEM images shown in Figure 2 (for a standard photo of the studied substrates refer to Figure S1 in Appendix I). The purpose of this study is to identify a substrate suitable for etextile electrodes based on $Z_{p}$. Please note, the correlation of $Z_{p}$ on the characteristics of the textile substrates is outside the scope of this study.

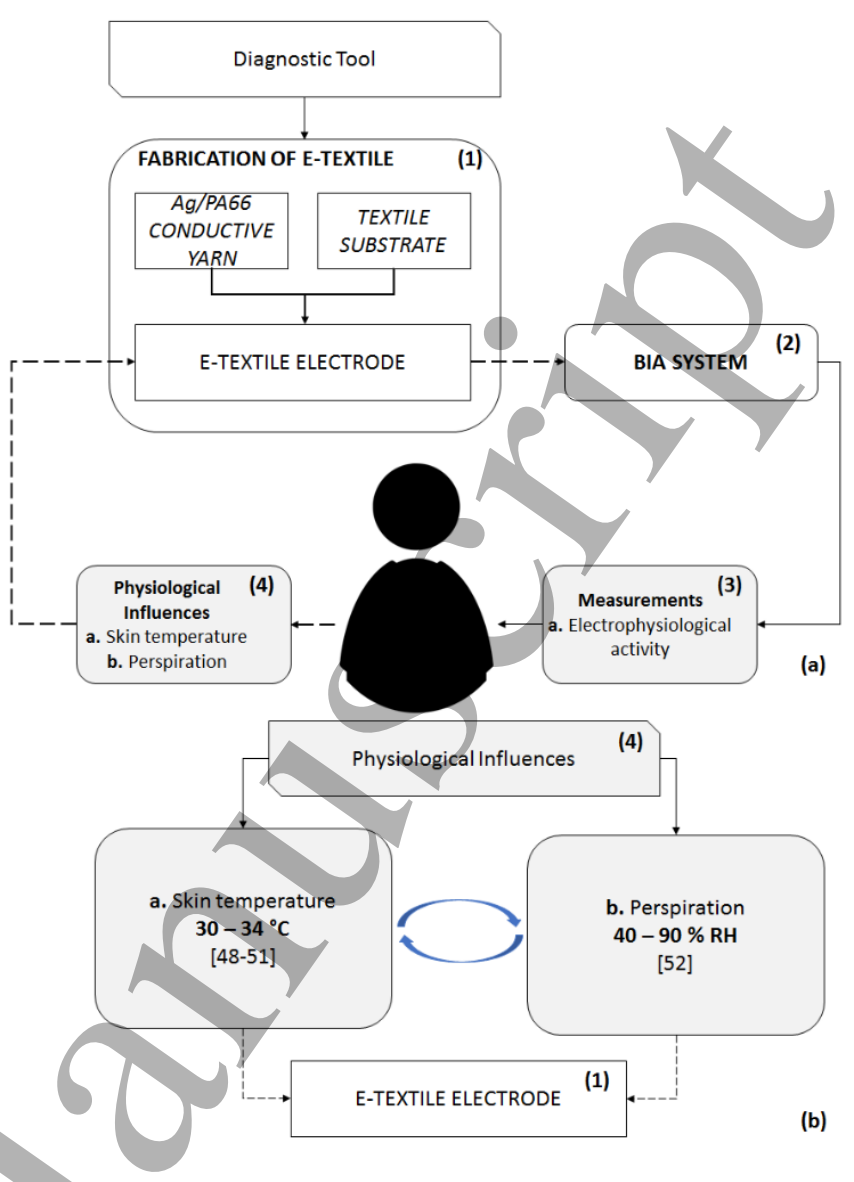

Figurê 1. Diagram showing connection between diagnostic tool and its constitutive components and patient: (a) connection between diagnostic tool and patient, (b) physiological influences on e-textile electrodes.

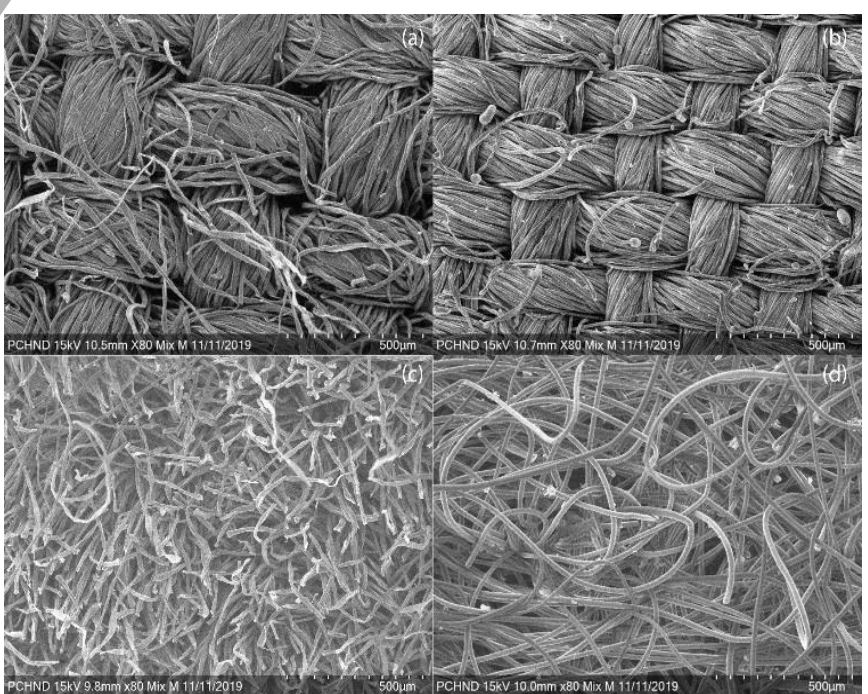

Figure 2. SEM images of substrates: (a) S1 (b) S2 (c) S3 and (d) S4. 
Table 1. Textile substrates used for e-textile electrodes.

\begin{tabular}{|c|c|c|c|c|}
\hline & $\begin{array}{l}\text { S1 for set of e-textile } \\
\text { electrodes } 1 \text { - E1 }\end{array}$ & $\begin{array}{l}\text { S2 for set of e-textile } \\
\text { electrodes } 2-\mathrm{E} 2\end{array}$ & $\begin{array}{l}\text { S3 for set of e-textile } \\
\text { electrodes } 3 \text { - E3 }\end{array}$ & $\begin{array}{l}\text { S4 for set of e-textile } \\
\text { electrodes } 4-\mathrm{E} 4\end{array}$ \\
\hline Description & Cotton Plain Weave & Cotton Poplin & Cotton Velvet & $\begin{array}{l}\text { Polyester Non-Woven } \\
\text { Felt }\end{array}$ \\
\hline Composition & Co $100 \%$ & Co $100 \%$ & Co $100 \%$ & PES $100 \%$ \\
\hline Structure & Plain Weave & Poplin & Velvet & Non Wover \\
\hline Weight $\left(\mathrm{g} / \mathrm{m}^{2}\right)$ & 200 & 100 & 300 & 200 \\
\hline $\begin{array}{l}\text { Thickness } \\
(\mathrm{mm})\end{array}$ & 0.65 & 0.4 & 1 & \\
\hline$\varepsilon_{r}$ & 1.12 & 1.14 & 1.33 & 1.20 \\
\hline
\end{tabular}

To fabricate the e-textile electrodes, we adapted the embroidery methodology reported in our previous study [35]. Shieldex Conductive Yarn - Silver Plated Nylon (Ag/PA66) 2x117/17 dtex (Shieldex U.S., Palmyra, NY, United States) was the conductive material possessing a linear resistance of about $1,500 \Omega / \mathrm{m}$. This was matched with a non-conductive $100 \%$ polyester (PES) thread based on relatively similar linear densities (specifically 29.5 and 39.8 tex) for the bobbin and needle thread, respectively (refer to Appendix I for textile units). To enable probes to connect to the e-textile electrodes, snap-fasteners were mechanically attached. The e-textile electrodes were designed on the EasyDesign EX software connected to the Singer Futura XL-550 embroidery machine. In our previous study we compared the influence of various embroidery characteristics on a single substrate. We identified that the e-textile electrodes measuring $40 \times 33$ $\mathrm{mm}$ with a stitch spacing (defined as density on the EasyDesign EX software) of $0.4 \mathrm{~mm}$ and a stitch length of $7 \mathrm{~mm}$ resulted in the lowest $Z_{p}$ [35]. When measuring $\mathrm{Zp}$ of the e-textile samples, the electrode is considered as a whole and the yarn is directly attached to the substrate. The density of the stitch selected from our previous study is indicative of a stable embroidered surface of the e-textile electrodes approximating an ideal homogeneous metallic electrode. A stable stitch density has been proven to be important in more restrictive electronic systems such as antennas where lossses and imperfections are extremelly critical as they operate at significantly higher frequencies than in our case [54-56]. Therefore, these variables were adapted for the embroidery process on the various substrates used in this study. Figure 3 presents the e-textile electrodes fabricated using the selected substrates depicted in Table 1. The size and shape of the e-textiles was controlled with the EasyDesign EX software. To fabricate the e-electrodes to required dimensions, initial samples were designed with the expected dimensions and by measuring the actual embroiered samples, we modified the dimensions on the software accordingly. This allowed us to resolve the small tolerance error associated with embroidery machines [57]. The samples of each set were weighed and the length was calculate using the textile units



Figure 3. E-textile electrodes on various substrates: (i) cotton plain weave (E1), (ii) cotton poplin (E2), (iii) cotton velvet (E3) and (d) polyester non-woven felt (E4).

units formula (refer to Appendix 1). The length of the threads for each sample set were relatively close to the first decimal place, where: E1, $7.9 \mathrm{~m}$; E2, $7.3 \mathrm{~m}$; E3 $7.7 \mathrm{~m}$ and E4: $7.6 \mathrm{~m}$. This slight variance can be associated with the differences in structure and thickness of the substrate. Provided the embroidery settings are consistent across all samples, the main difference between the e-textile electrodes is due to the substrate, considering the electrode as a whole. Therefore, any differences between the sample sets due to the amount of conductive thread or the threads contact with the substrate is related to the substrate itself. This could be due to the thickness or the surface roughness of the substrate. Thus, a selection of the substrate is made based on the results of its efficiency as an e-textile electrode.

\subsection{Experimental Setup}

We adapted the triple-layer wafer testing method from our previous study [35] to identify $Z_{p}$ of the various etextile electrodes. Two identical electrodes were sandwiched together, one positioned on the top and the other on the bottom with the conductive sides interfacing an interposed polyester swatch (measuring $45 \times 40 \mathrm{~mm}$ ), 
acting as an insulator to avoid a short circuit from the electrode snaps, refer to Fig. 4. Polyester is impervious to water and does not absorb humidity; therefore, this textile is ideal as a dielectric material due to its consistent electrical impedance over time, since moisture does not change $\varepsilon_{r}$ for hydrophobic fabrics.

Tests were conducted using a bipolar electrode setup, with two leads connecting to a voltage driven HM8118 LCR Bridge/Meter (Rohde \& Schwarz, Munich, Germany - accuracy: $0.05 \%$ ) set at a frequency of $50 \mathrm{kHz}$. The electrodes were stabilised at each corner with nonconductive wooden craft pegs. They were carefully positioned at the outer-edge of the electrode (coming into contact with its substrate) avoiding any pressure distribution across the actual electrode surface which can impact the measurements as described in our previous study [35].

This setup was adapted with the electrodes placed in the climatic chamber by passing the leads connected to the LCR bridge through the aperture of the climatic chamber that was closed with a rubber plug as a seal. The nonconductive wooden craft pegs rested on the grill of the climatic chamber maintaing the e-textile electrodes at a distance from the grill avoiding any electrical interference. We used this equipment to measure the resistance $(R)$ and reactance $\left(X_{C}\right)$ of the material and then calculated $Z_{p}$ of the e-textile using the following equation:

$$
\left|Z_{p}\right|=\sqrt{R^{2}+X_{C}^{2}}
$$

$$
\text { where } X_{C}=\frac{1}{2 \pi f C} \text {. }
$$

For the remainder of the manuscript, we have used $Z_{p}$ as the modulus for simplicity. This study is based on a preceding investigation of the properties of textiles demonstrating a small variation of resistance from one sample set to another using the same textile substrate which indicated that textiles have a reasonably well-defined resistivity [58]. The measurements were conducted as a continuous function of time, referred to as dynamic measurements, where $Z_{p}$ remained consistent over a three minute period. Thus, the use of standard deviation and error bars are not essential for these measurements [35]. Therefore, in this study, the e-textile electrodes were evaluated using the identical methodology, the first set of tests was conducted at a room temperature of $25^{\circ} \mathrm{C}$.

To study the influence of temperature and moisture on $Z_{p}$ of the e-textile electrodes, we placed the electrodes in a CCK-25/48 climatic chamber (Dycometal, Spain). The temperatures tested ranged from 30 to $34^{\circ} \mathrm{C}$, and for each temperature setting we increased $\mathrm{RH}$ from 40 to $90 \%$ at $10 \%$ intervals emulating skin properties, refer to Figure 4 (with reference to Section 1.1 and Fig. 1). By using a climatic chamber, the skin wetness boundaries defined by Mole et al. [52] is transposed to moisture content of the etextile electrodes. The samples were placed in the climatic chamber for a set temperature and the RH set and the LCR bridge monitored $Z_{p}$ for a duration of three minutes. Following this period, the RH was increased by $10 \%$. During the transient periods before executing the experiments for the new temperature setting, the electrodes were taken out of the climatic chamber and let to dry. Air permeability of the electrodes tests were conducted using PROWHITE AIRTEST II (PROWHITE, Istanbul, Turkey) set at $200 \mathrm{~Pa}$. We initially measured the air permeability of the various substrates before measuring the air permeability of the e-textile electrodes [59] fabricated with these different substrates.



Figure 4. Experimental setup emulating skin temperature and perspiration by: (a) theoretical correlation of skin parameters to climatic chamber and (b) climatic chamber experimental protocol. 


\section{Results}

\section{1 $Z_{p}$ of e-textile electrodes in ambient conditions}

By using various textile substrates for the e-textile electrodes at room temperature, it is expected for $Z_{p}$ to differ. In fact, due to the electrode capacitor configuration made with the parallel metallic layers, the difference in relative permittivity of the various textile substrates impacted the overall impedance. $Z_{p}$ for the e-textile electrode sets E1, E2, E3 and E4 were measured producing $269,284,258$ and $244 \mathrm{k} \Omega$ respectively.

The polyester non-woven felt e-textile electrode (E4) demonstrated the lowest $Z_{p}$ contrary to the cotton poplin etextile electrode (E2) resulting in a large $Z_{p}$, with a relative difference of about $15 \%$. Taking into account the consistency of $Z_{p}$ in e-textiles [35], this implies that the polyester non-woven textile is the most suitable as a substrate for use at room temperature. Further tests were conducted to identify the impact of temperature and moisture on $Z_{p}$ for the e-textile electrodes relative to their substrate.

As can be seen from Fig. 5, there is no direct correlation between $Z_{p}$ and any of the properties relating to the textile substrates presented in Table $1 . Z_{p}$ can be influenced by other textile parameters, i.e. the linear density of the thread (cotton or polyester thread), the structure of the textile (plain weave, poplin, velvet or non-woven), or any finishing done on the textile [60], which are outside of the scope of this paper.

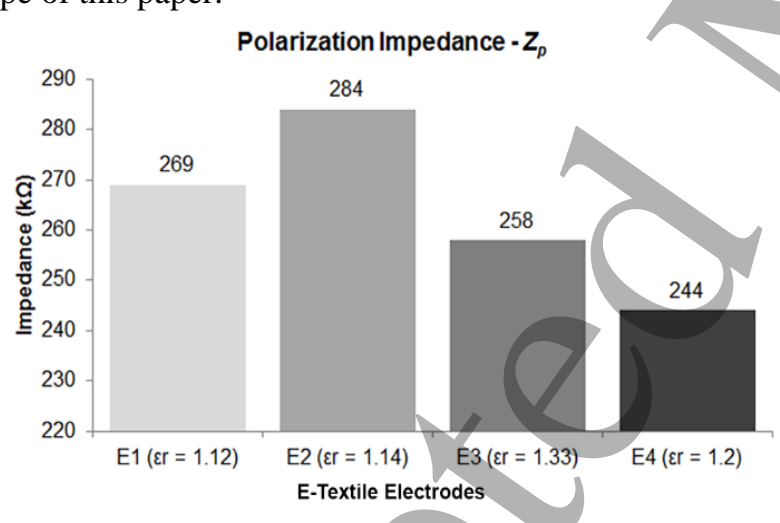

Figure 5. $Z_{p}$ of e-textile electrodes at room temperature $25^{\circ} \mathrm{C}$ presented with $\varepsilon_{\mathrm{r}}$ of substrate.

\section{2 $Z_{p}$ of e-textile electrodes in climatic chamber}

Fig. 6 presents presents the influence of temperature and RH variances on $Z_{p}$. The obtained results reveal that there is no direct correlation between $Z_{p}$ and changes in temperature for any of the e-textile electrodes (for absolute values refer to Table S1, Appendix II). To illustrate further the behaviour of $Z_{p}$ relative to temperature, we plotted the change in $Z_{p}$ relative to the individual temperature increments. Referring to Fig. 7, it is evident that the influence of temperature on $Z_{p}$ is inconsistent. For each $\mathrm{RH}$, $Z_{p}$ increases and decreases as the temperature increases by $1^{\circ} \mathrm{C}$. This is true across all RH/settings for each sample set and across all the sets of samples. However, there are some instances where there is no change. This is prevelant in samples E4. Samples E1 demonstrates the greatest variance relative to an increase in temperature contrary to samples E2 and E3. Samples E4 indicates relatively larger changes in $Z_{p}$ close to the boundaries, across all RH. For example, an increase in temperature from $30^{\circ} \mathrm{C}-31^{\circ} \mathrm{C}$ and $33^{\circ} \mathrm{C}-34^{\circ} \mathrm{C}$ with the least changes occurring from $31^{\circ} \mathrm{C}-33^{\circ} \mathrm{C}$. It is also evident that $\mathrm{RH}$ influences $Z_{p}$. Table 2 presents the relative difference between $Z_{p(T \max )}$ and $Z_{p(\text { Tmin })}$ across the thermophysiological skin temperature boundaries $\left(30^{\circ} \mathrm{C}\right.$ $34^{\circ} \mathrm{C}$ ) relating to the individual $\mathrm{RH}$, calculated using Equation 3:

$$
\Delta Z_{p}(\%)=\frac{Z_{p(\text { Tmax })}-Z_{p(\text { Tmin })}}{Z_{p(\text { Tmin })}} \times 100
$$

It is clear that $Z_{p}$ is impacted by change in RH. Thus, we also plotted the change in $Z_{p}$ relative to the $10 \% \mathrm{RH}$ increments against the temperature settings, shown in Fig. 8.

It can be seen from Fig. 8 that the increase in RH induces a greater change in $Z_{p}$ compared to the effect of different temperatures. However, there is inconsistency across RH relative to the temperature increments across all samples. By applying polynomial trendlines on this data, for a substantial amount of trends $\mathrm{R}^{2}<0.5$. This is particularly true of samples E1. Samples E2, E3 and E4 are indicative of a stronger $\mathrm{R}^{2}$ yet fail to satisfy any mathematical relation across the equations, for example the parabola alternates between positive and negative, is slightly wide or slightly narrow; thus, disproving any relation across temperature (please refer to Table S2, Appendix II). A significant drop in $Z_{p}$ occurrs at $80-90 \% \mathrm{RH}$ across samples E1, E2 and E3 while this is also evident in sample $\mathrm{E} 4$, it is relatively small in comparison. 
Cotton Plain Weave E-Textile Electrodes (E1)

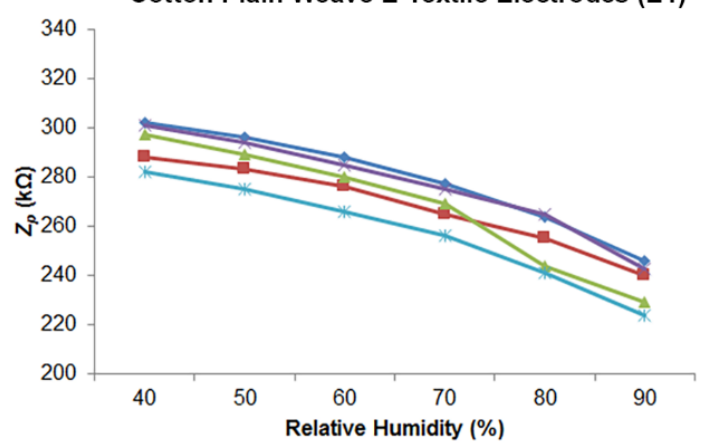

Cotton Velvet E-Textile Electrodes (E3)

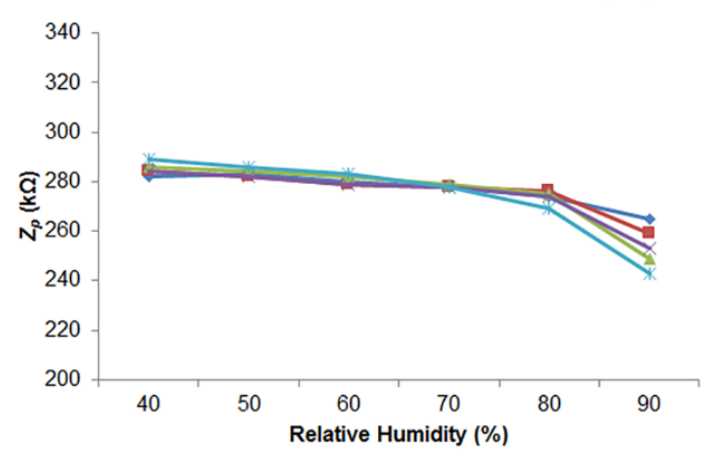

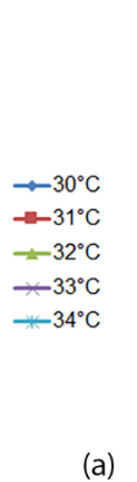
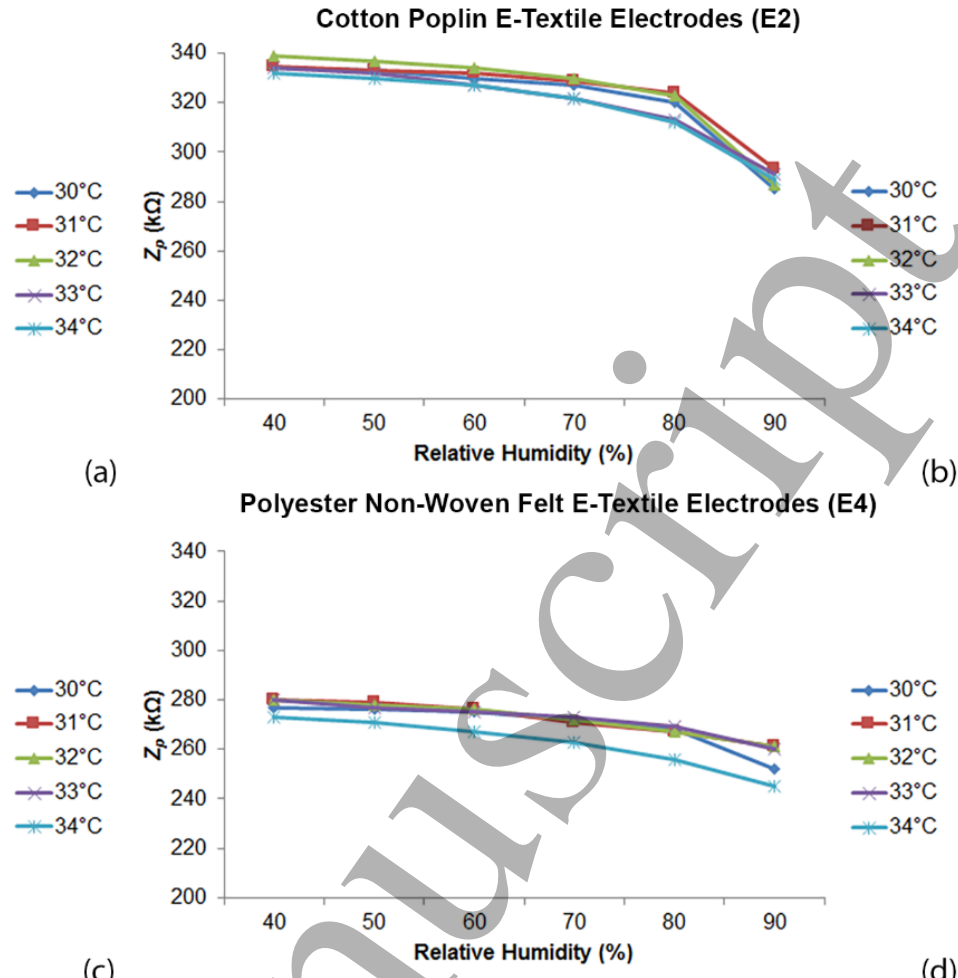

Polyester Non-Woven Felt E-Textile Electrodes (E4)

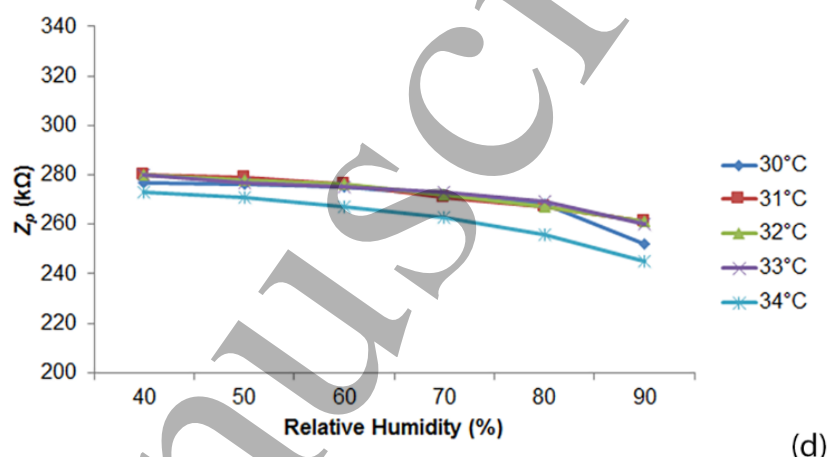

Figure 6. Influence of skin temperature and skin wetness (RH) on $Z_{p}$ for e-textile electrodes (a) E1 [61], (b) E2, (c) E3 and (d) E4 [61].

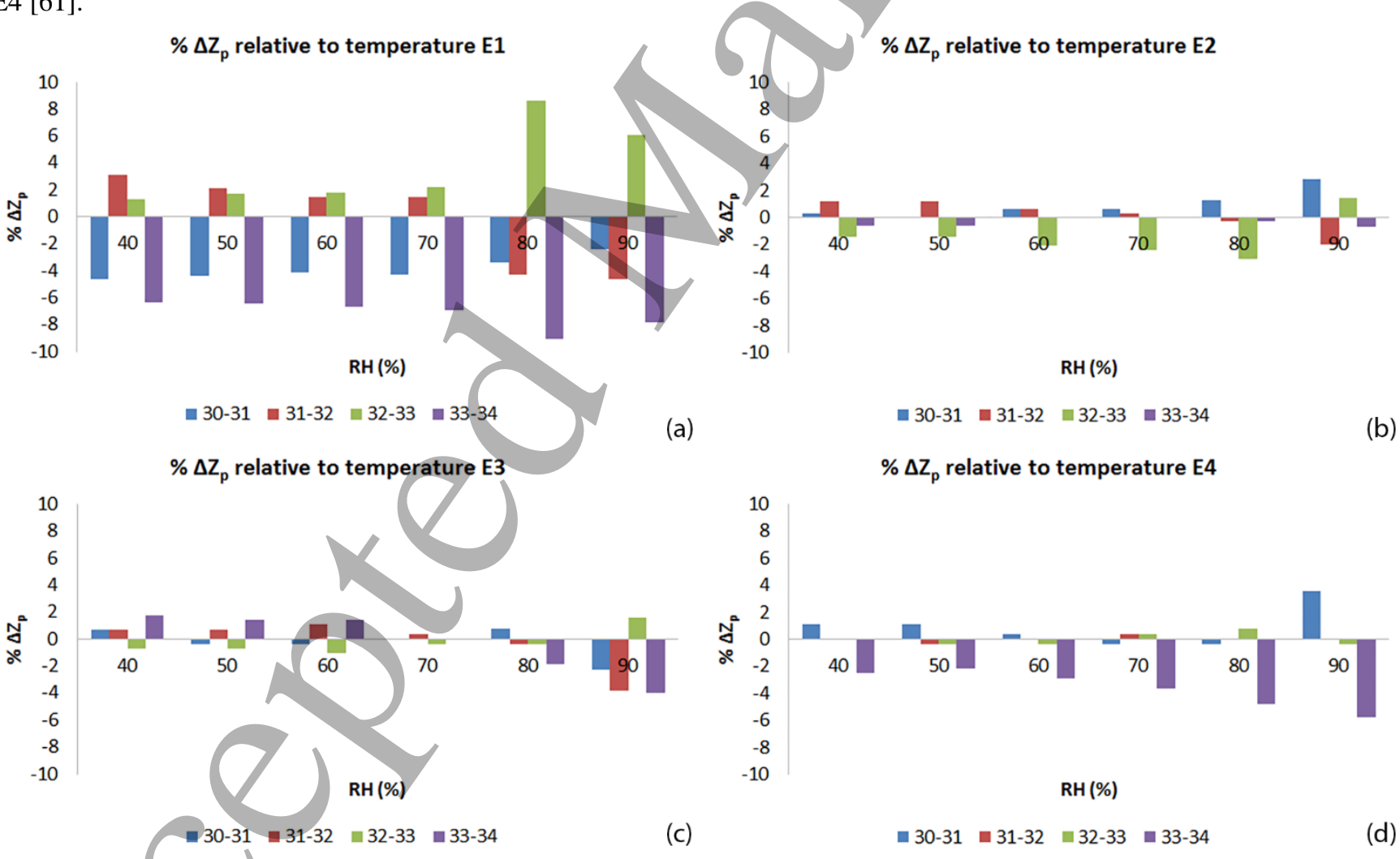

Figure 7. Change in $\mathrm{Z}_{\mathrm{p}}$ relative to temperature. 
Table 2. Relative difference of $Z_{p}$ across thermophysiological skin temperature boundaries relative to RH.

\begin{tabular}{|c|c|c|c|c|c|c|c|c|c|c|c|c|}
\hline & $40 \%$ & $50 \%$ & $60 \%$ & $70 \%$ & $80 \%$ & $90 \%$ & $40 \%$ & $50 \%$ & $60 \%$ & $70 \%$ & $80 \%$ & $90 \%$ \\
\hline & \multicolumn{6}{|c|}{ E1 } & \multicolumn{6}{|c|}{ E2 } \\
\hline \multirow[t]{2}{*}{$\Delta Z_{p}{ }^{*}(\%)$} & 7.1 & 7.6 & 8.2 & 8.2 & 9.9 & 9.8 & 2.1 & 2.1 & 2.1 & 2.4 & 3.8 & 2.8 \\
\hline & \multicolumn{6}{|c|}{ E3 } & \multicolumn{6}{|c|}{ E4 } \\
\hline$\Delta Z_{p}^{*}(\%)$ & 2.4 & 1.4 & 1.4 & 0.4 & 2.6 & 9.0 & 2.5 & 2.9 & 3.3 & 3.8 & 5.0 & 6.5 \\
\hline
\end{tabular}

*Relative difference of $Z_{p}$ across temperature range

$\mathrm{E} 1 \% \Delta \mathrm{Z}_{\mathrm{p}}$ relative to temperature against $\mathrm{RH}$



$\mathrm{E} 3 \% \Delta \mathrm{Z}_{\mathrm{p}}$ relative to temperature against $\mathrm{RH}$

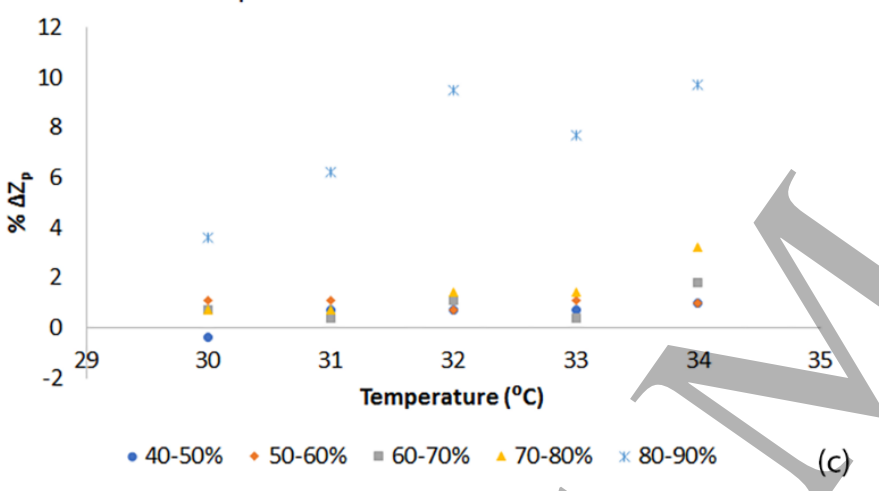

Figure 8. Change in $\mathrm{Z}_{\mathrm{p}}$ relative to $\mathrm{RH}$ increments.

Summarising these findings, we suggest that the impact of temperature on $Z_{p}$ is dependent on $\mathrm{RH}$. For example, the increase in $\mathrm{RH}$ significantly affected $Z_{p}$, specifically at $90 \% \mathrm{RH}$. This implies that $Z_{p}$ depends on both temperature and RH. However, observing the changes in $Z_{p}$ for each sample set (as shown in Fig. 6), a correlation can be seen for individual temperature settings against RH. It is evident that by increasing RH the e-textile electrodes become wetter and $Z_{p}$ decreases.

As shown in Fig. 8, the significant decrease in $Z_{p}$ at $90 \%$ $\mathrm{RH}$ occurs across all electrodes. However, this is expected as at about $80 \%$ RH the textile begins to dampen, meaning that at $90 \% \mathrm{RH}$ the moisture content has caused sufficient dampening of the substrate. $Z_{p}$ displays a substantial drop between 80 and $90 \% \mathrm{RH}$, thus we were interested to study the effect of $\mathrm{RH}$ in the range of $40-80 \%$ on $Z_{p}$. For this, we adapted linear regression to the results presented in Fig 6 . By observing the slope $(m)$ of the linear regression $(y=m x$
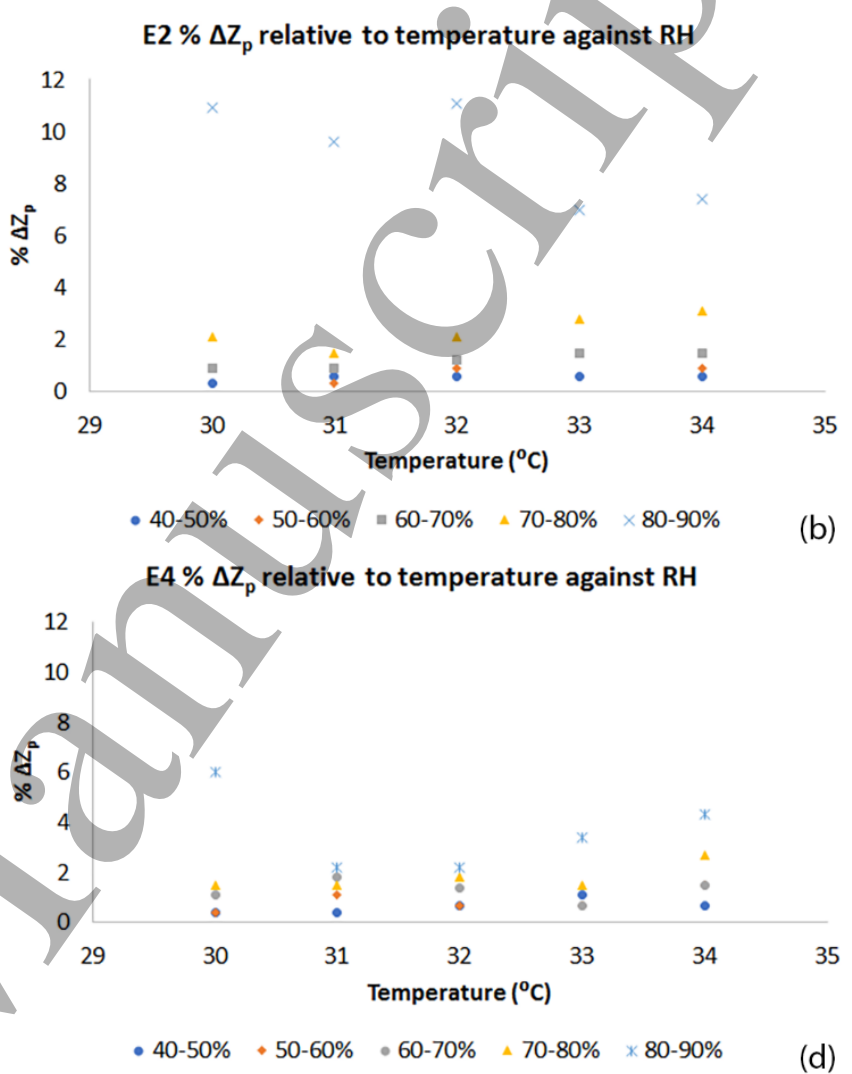

$+c$ ), as shown in Fig 9, relating to the various e-textile electrode samples (Table 3 ), it is noticeable that $Z_{p}$ of samples E3 and E4 are impacted the least by changes in temperature and $\mathrm{RH}$ at $40-80 \%$. This is evident by the least variation in slope across all temperatures.

Provided that both E3 and E4 are the closest to representing a "zero slope" indicating a small change, E4 is preferred as it generally possesses a lower y intercept. Thus, we have the advantage of a small change due to skin temperature and perspiration while maintaining a lower $Z_{p}$. This is ideal for electrophysiological measurements as influences deriving from changes in skin temperature and humidity variations from perspiration can cause instability in BIA measurements. In addition, by determining the influence of temperature and humidity on various substrates, this scale of interference can be accounted for in the calibration design of the system. 
Table 3. Characterization of linear regressions across skin temperature boundaries relative to $40-80 \% \mathrm{RH}: \mathrm{y}=\mathrm{mx}+\mathrm{c}$.

\begin{tabular}{ccccccccccc}
\hline & $\mathbf{3 0}^{\circ} \mathbf{C}$ & $\mathbf{R}^{\mathbf{2}}$ & $\mathbf{3 1}^{\circ} \mathbf{C}$ & $\mathbf{R}^{\mathbf{2}}$ & $\mathbf{3 2}^{\circ} \mathbf{C}$ & $\mathbf{R}^{\mathbf{2}}$ & $\mathbf{3 3}^{\circ} \mathbf{C}$ & $\mathbf{R}^{\mathbf{2}}$ & $\mathbf{3 4}^{\circ} \mathbf{C}$ & $\mathbf{R}^{\mathbf{2}}$ \\
\hline$E 1$ & $-1.0 x+342$ & 0.98 & $-0.8 x+234$ & 0.98 & $-1.3 x+351$ & 0.93 & $-0.9 x+339$ & 0.99 & $-1.0 x+325$ & 0.98 \\
$E 2$ & $-0.3 x+349$ & 0.91 & $-0.3 x+346$ & 0.92 & $-0.4 x+356$ & 0.94 & $-0.5 x+357$ & 0.95 & $-0.5 x+297$ & 0.90 \\
$E 3$ & $-0.2 x+292$ & 0.86 & $-0.2 x+292$ & 0.98 & $-0.3 x+297$ & 0.97 & $-0.2 x+293$ & 0.97 & $-0.5 x+310$ & 0.94 \\
$E 4$ & $-0.2 x+287$ & 0.91 & $-0.3 x+295$ & 0.95 & $-0.3 x+294$ & 0.96 & $-0.2 x+290$ & 0.98 & $-0.4 x+291$ & 0.96 \\
\hline
\end{tabular}
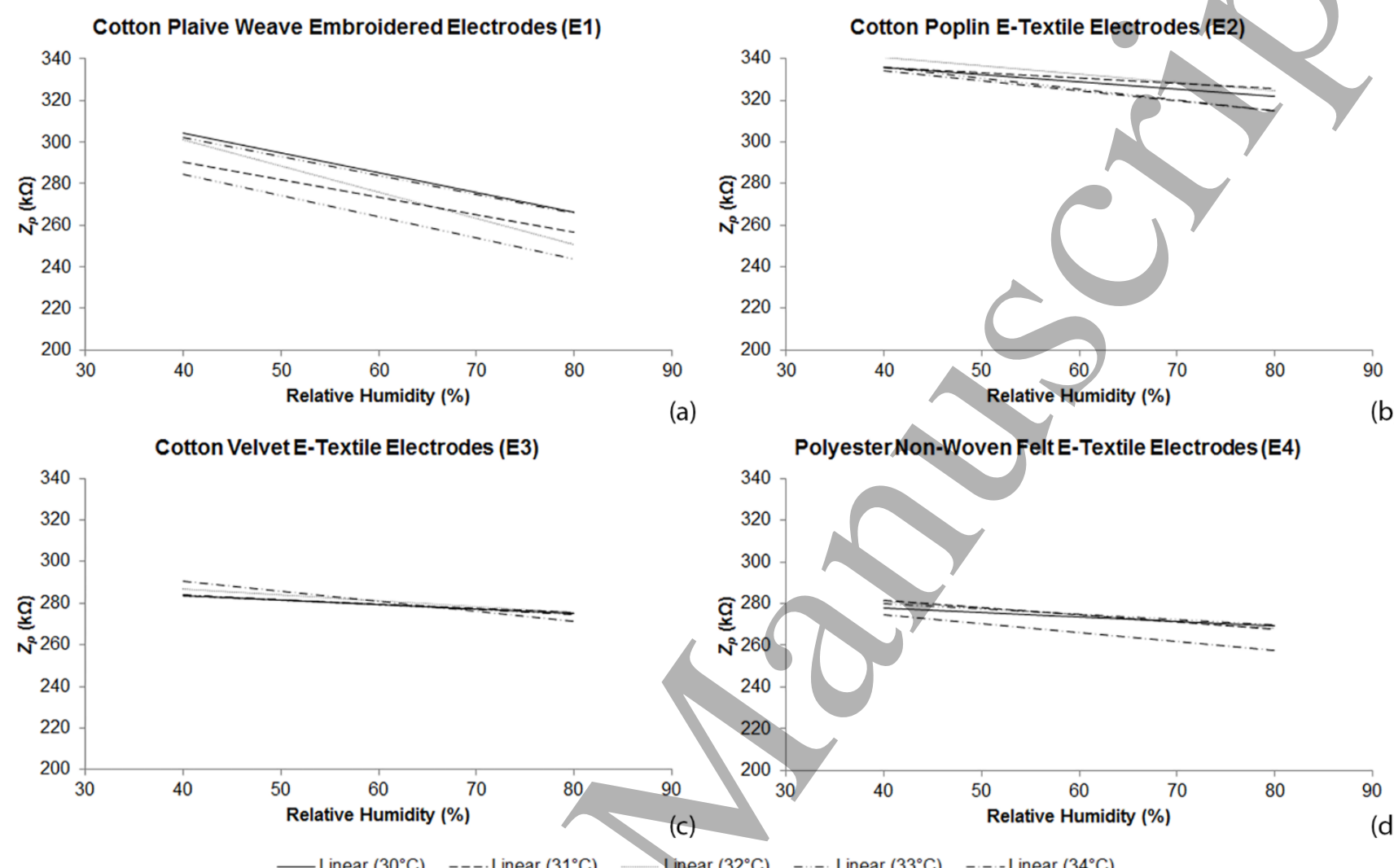

(a)

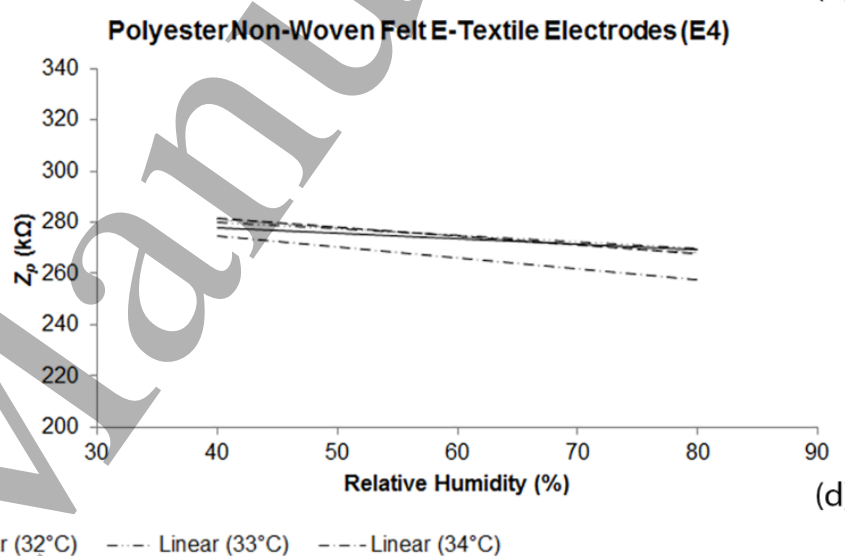

(b)

Figure 9. Linear regression of $Z_{p}$ due to skin temperature and skin wetness 40 - $80 \%$ RH for samples: (a) E1, (b) E2, (c) E3 and (d) E4.

\subsection{Air permeability of textile substrates and e- textile electrodes}

The air permeability tests for the selected e-textiles were conducted to identify the e-textile electrode suitable relative to the physiological comfort associated with textiles. To ensure the consistency of embroidery across the samples, SEM images of e-textile electrodes are presented in Fig. 10. By testing the air permeability of the textile substrates (S1, S2, S3, S4) prior to the fabrication process, results show the polyester non-woven felt (S4) had the largest air permeability. For the fabricated e-textile electrodes (E1, E2, E3 and E4), a reduction in air permeability was expected due to the extra two layers of Ag/PA66 and PES stitching (Fig. 11). Thus, even with

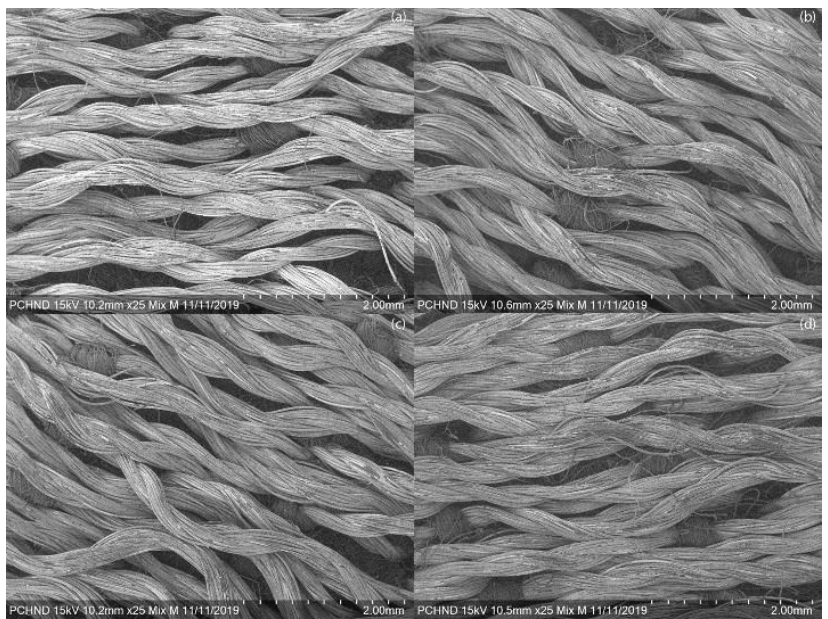

Figure 10. SEM images of e-textile electrodes (a) E1, (b) E2, (c) E3 and (d) E4. 


\section{Air Permeability of Substrate}



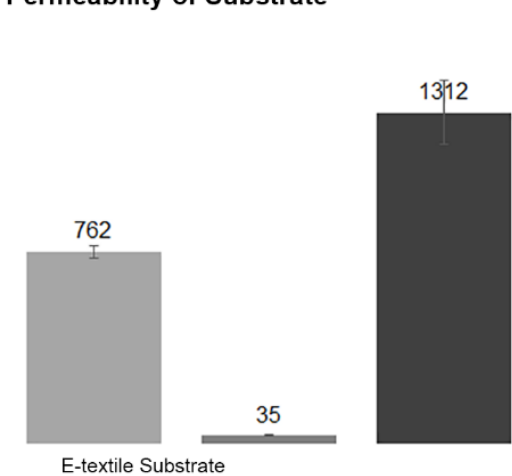

E-textile Substrate
Air Permeability of E-Textile Electrodes

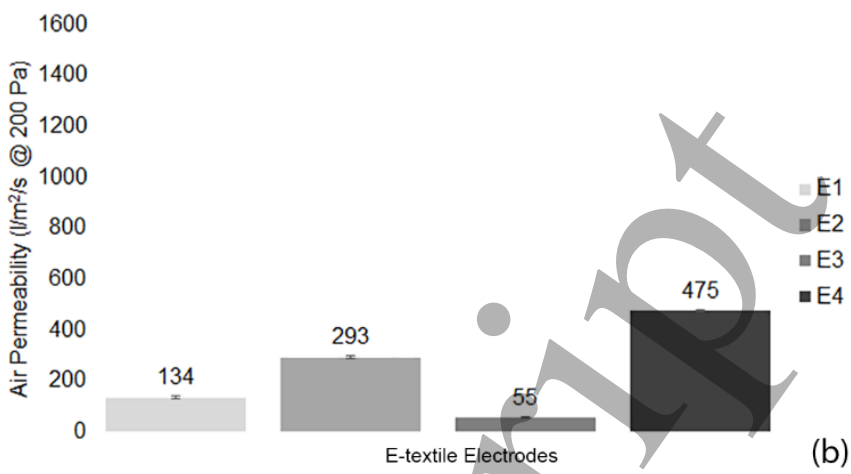

Figure 11. Air permeability of (a) textile substrates and (b) e-textile electrodes.

these extra layers the substrate S4 used to fabricate E4 permits more air to flow through the electrodes compared to E1, E2 and E3. To conclude, E4 is less affected by changes to the thermal properties of skin while maintaining physiological comfort for the subject in comparison to the other substrates used in this study.

\section{Discussion}

Our e-textile electrodes comprise of three materials: (i) the substrate, (ii) PES thread and (iii) Ag/PA66 thread. Each material possesses its own material characteristics. The cotton and polyester substrates ( 1, S2, S3 and S4), including the PES thread are considered insulators, while Ag/PA66 possesses silver electrolytes and is primarily responsible for the presence of $Z_{p}$ in the e-textile electrodes. However, provided that the amount of conductive yarn present in each e-textile electrode is relatively close, it is evident that the substrate selection has an influence on $Z_{p}$ of e-textile electrodes.

There appears to be no distinguishable relationship between $Z_{p}$ and the various $\varepsilon_{r}$ of the substrates used in the e-textile electrodes for this study. For example, E2 and E4 exhibit the highest and lowest $Z_{p}$ (284 and $244 \mathrm{k} \Omega$, respectively) while their measured $\varepsilon_{r}(1.14$ and 1.20) fall between the highest and lowest permittivities of the substrates used for E1 and E3 (1.12 and 1.33, respectively). This could be attributed to the electrolyte impurities found in the substrate. It is feasible that these unidentified electrolytes contribute to the $Z_{p}$ characterization relative to temperature and RH. Our findings show there was no direct correlation of $Z_{p}$ relative to temperature. Contrary, transposing skin wetness into moisture/content it was identified that $Z_{p}$ decreases as $\mathrm{RH}$ increases across all etextile electrodes.

Murphy et al. [58] demonstrated the electrolyte impurities found in cotton textiles cause a decrease in resistance relative to an increase in temperature and moisture content (RH). It was also shown that for a RH greater than about $80 \%$ the cotton textiles undergo polarization indicating an increase in conductance. Electrolyte impurities found in polyester also create a small conductance in the substrate. Shahzad et al. [62] fabricated polyester and stainless-steel hybrid conductive thread (PSS). Testing the resistance of the threads in a climatic chamber indicated that a PSS thread pertains a close to constant resistivity relative to an increase in RH up to $80 \%$. This resistivity decreases when RH reaches over $80 \%$.

Murphy et al. and Shahzad et al. [58, 62] demonstrated the resistance of materials independently using direct current measurements. Although our research combines conductive and insulating materials using alternating current (AC) at a predefined frequency resulting in resistance and reactance measurements, our results are in agreement with the findings of Murphy et al. and Shahzad et al. $[54,58]$. The fabrication of e-textile electrodes gives rise to direct contact of the independent materials creating a distinct functional material resulting in the interaction of various electrolyte impurities in addition to the strong presence of silver electrolytes in the Ag/PA66 thread.

The distilled water supplying climatic chambers contains electrolyte impurities enhancing the conductivity $[63,64]$. This can contribute to the reactions of the impurities in the three different materials used to fabricate the e-textile electrodes. The behaviour and reaction of these impurities may be the cause of changes in $Z_{p}$ demonstrating no correlation relative to temperature, as opposed to the study conducted by Murphy et al. [58]. However, our research relating $Z_{p}$ to $\mathrm{RH}$ is consistent with the research undertaken by Murphy et al. and Shahzad et al. [58, 62]. Shahzad et al.'s PSS is high in electrolytic conductivity (about $1,300 \Omega / \mathrm{m}$ linear resististance) analogous to $\mathrm{Ag} / \mathrm{Pa} 66$, therefore a close to constant resistivity of this thread is expected with a decrease at about $80 \% \mathrm{RH}$ across all e-textile electrodes. Hence, the decrease in $Z_{p}$ between $40-80 \% \mathrm{RH}$ could be from the reaction of the distilled water with the impurities of the substrates and PES thread. As polyester has a moisture regain of $0.4 \%$ while cotton is about $8.5 \%$, it is expected for the polyester non-woven felt 
e-textile electrodes to demonstrate a closer to zero gradient in comparison to the cotton e-textile electrodes. While the greater drop in $Z_{p}$ occurring at $90 \%$ is also attributed to reaction of the Ag/PA66 electrolytic conductivity.

To further tailor this research, a solution imitating human perspiration needs to be used with the climatic chamber. For example, perspiration is a combination of minerals, lactic acid, and urea. The minerals include sodium, potassium, calcium, and magnesium. These minerals are all conductive elements while lactic acid has slight conductive properties as opposed to urea which is an insulator. Due to the complexity of the electrolytes present in all materials, in addition to the substances found in perspiration, repeatability will be conducted over an hourly period to gain a more accurate insight into the influence of sweat on $Z_{p}$ which in turn impacts $Z_{e s}$. This time period is significant as the conductivity of electrolytes in impurities can vary over time. Moreover, adapting the electrodes to a BIA system, the signal-to-noise ratio (SNR) can be determined for the various textiles.

Previous research into e-textile electrodes have addressed various stitch characteristics, signal-to-noise ratio (SNR) analysis and direct comparison of $\mathrm{Ag} / \mathrm{AgCl}$ to e-textile electrodes in ECG and EMG applications [16, 18]. These studies were based on a single substrate applied on healthy subjects, thus neglecting to consider the impact of various substrates in addition to varying physiological parameters of the skin as contributing factors. The findings of this study reveal the importance of selecting a suitable substrate for e-textile electrodes. Specifically, its influence on $Z_{p}$ when skin parameters change due to medical conditions. These are important factors when designing IoWT.

\section{Conclusion}

This study demonstrates the importance of selecting an appropriate substrate when designing e-textile electrodes. We show that skin temperature and perspiration influence $Z_{p}$ of these electrodes. The temperature emulating skin temperature demonstrated an inconsistent influence on $Z_{p}$. However, an increase in moisture content of the e-textile electrodes relative to an increase in $\mathrm{RH}$ resulted in a reduced $Z_{p}$. We identified polyester non-woven felt etextile electrodes as the most suitable for BIA systems to monitor electrophysiological activities while characterizing its trend relative to changes in skin properties. It has a relatively low $Z_{p}$ while exhibiting a linear relationship across the thermophysiological skin temperature ranges and perspiration. Moreover, its linear relationship approaching a 'zero-slope' indicates the little impact of skin parameters on $Z_{p}$. In addition, it possessed the highest air permeability supporting its suitability for wearable non-invasive biopotential electrodes. E-textile electrodes present a promising solution for long-term electrophysiological monitoring. However, many factors need to be evaluated before adapting them to BIA systems, such as physiological properties and material characteristics. A substudy of this research to be conducted includes assessing the dielectric properties of new textile materials in order obtain the $Z_{p}$ function in terms of their relative permittivity and dielectric losses with the aim of achieving novel ultra-low impedance electrodes. Moreover, future work can also include an extension of the sample set by comparing textile substrates with identical and distinct relative permittivities. This work forms the foundation for future studies on the practicality of e-textile electrodes and their impact on $Z_{e s}$ influencing $Z_{m}$ in order to obtain $Z_{B U S}$, while taking into consideration $N$ [40-43]. To measure $Z_{m}$, tests will be conducted on pork belly emulating human skin in terms of its dielectric properties, skin thickness and underlying biotissue structure. Pork belly as a biotissue allows for replicates and repeatability, thus an accurate insight/into e-textile electrodes can be explored. An objective BIA diagnostic tool will enable home monitoring of a patient's electrophysiological conditions, thus improving the quality of health care for a patient in addition to a reduced burden on the health care system.

\section{Acknowledgements}

This work was supported by the Spanish Government MINECO under Project TEC2016-79465-R.

\section{References}

[1] Nescolarde L, Yanguas J, Terricabras J, Lukaski H, Alomar X, Rosell-Ferrer J and Rodas G 2017 Detection of muscle gap by L-BIA in muscle injuries: clinical prognosis Physiol Meas 38 L1L9

[2] Nescolarde L, Yanguas J, Lukaski H, Alomar X, Rosell-Ferrer J and Rodas G 2013 Localized bioimpedance to assess muscle injury Physiol Meas 34 237-45

[3] Nescolarde L, Yanguas J, Lukaski H, Alomar X, Rosell-Ferrer J and Rodas G 2015 Effects of muscle injury severity on localized bioimpedance measurements Physiol Meas 36 27-42

[4] Harrison A P, Elbrond V S, Riis-Olesen K and Bartels E M 2015 Multi-frequency bioimpedance in equine muscle assessment Physiol Meas 36 453-64

[5] Cumming K, Hoyle G E, Hutchison J D and Soiza R L 2014 Bioelectrical impedance analysis is more accurate than clinical examination in determining the volaemic status of elderly patients with fragility fracture and hyponatraemia $J$ Nutr Health Aging 18 744-50 
[6] Dean D A, Ramanathan T, Machado D and Sundararajan R 2008 Electrical Impedance Spectroscopy Study of Biological Tissues $J$ Electrostat 66 165-77

[7] Kyle U G, Bosaeus I, De Lorenzo A D, Deurenberg P, Elia M, Gomez J M, Heitmann B L, Kent-Smith L, Melchior J C, Pirlich M, Scharfetter H, Schols A M, Pichard C and Composition of the E W G 2004 Bioelectrical impedance analysis--part I: review of principles and methods Clin Nutr 23 1226-43

[8] Kyle U G, Bosaeus I, De Lorenzo A D, Deurenberg P, Elia M, Manuel Gomez J, Lilienthal Heitmann B, Kent-Smith L, Melchior J C, Pirlich M, Scharfetter H, A M W J S, Pichard $\mathrm{C}$ and Espen 2004 Bioelectrical impedance analysis-part II: utilization in clinical practice $\mathrm{Clin}$ Nutr 23 1430-53

[9] Baghbani R, Moradi M H and Shadmehr M B 2018 The Development of a Four-Electrode BioImpedance Sensor for Identification and Localization of Deep Pulmonary Nodules Ann Biomed Eng 46 1079-90

[10] Khalil S F, Mohktar M S and Ibrahim F 2014 The theory and fundamentals of bioimpedance analysis in clinical status monitoring and diagnosis of diseases Sensors (Basel) 14 10895928

[11] King R J, Clamp J A, Hutchinson J W and Moran C G 2007 Bioelectrical impedance: a new method for measuring post-traumatic swelling $J$ Orthop Trauma 21 462-8

[12] Lukaski H C and Moore M 2012 Bioelectrical impedance assessment of wound healing $J$ Diabetes Sci Technol 6 209-12

[13] Swisher S L, Lin M C, Liao A, Leeflang E J, Khan Y, Pavinatto F J, Mann K, Naujokas A, Young D, Roy S, Harrison M R, Arias A C, Subramanian V and Maharbiz M M 2015 Impedance sensing device enables early detection of pressure ulcers in vivo Nat Commun 66575

[14] Lukaski H 1996 Biological indexes considered in the derivation of the bioelectrical impedance analysis The American journal of clinical nutrition 64 397S-404S

[15] Piwek L, Ellis D A, Andrews S and Joinson A 2016 The Rise of Consumer Health Wearables: Promises and Barriers PLoS Med 13 e1001953

[16] Kannaian T, Neelaveni $R$ and Thilagavathi G 2012 Design and development of embroidered textile electrodes for continuous measurement of electrocardiogram signals Journal of Industrial Textiles 42 303-18

[17] Goncu Berk G 2018 Design of a wearable pain management system with embroidered TENS electrodes International Journal of Clothing Science and Technology 30 38-48
[18] Shafti A, Manero R B R, Borg A M, Althoefer K and Howard M J 2016 Designing embroidered electrodes for wearable surface electromyography. In: 2016 IEEE International Conference on Robotics and Automation (ICRA), pp 172-7

[19] Weder M, Hegemann D, Amberg M, Hess M, Boesel L F, Abacherli R, Meyer V R and Rossi R M 2015 Embroidered electrode with silver/titanium coating for long-term ECG monitoring Sensors (Basel) 15 1750-9

[20] Yapici M K, Alkhidir T, Samad Y A and Liao K 2015 Graphene-clad textile electrodes for electrocardiogram monitoring Sensors and Actuators B: Chemical 221 1469-74

[21] Yapici M K and Alkhidir T E 2017 Intelligent Medical Garments with Graphene-Functionalized Smart-Cloth ECG Sensors Sensors (Basel) 17

[22] Bihar E, Roberts T, Ismailova E, Saadaoui M, Isik M, Sanchez-Sanchez A, Mecerreyes D, Hervé T, De Graaf J B and Malliaras G G 2017 Fully Printed Electrodes on Stretchable Textiles for Long-Term Electrophysiology Advanced Materials Technologies 21600251

[23] Takamatsu S, Lonjaret T, Crisp D, Badier J M, Malliaras G G and Ismailova E 2015 Direct patterning of organic conductors on knitted textiles for long-term electrocardiography Sci Rep 515003

[24] Karim N, Afroj S, Malandraki A, Butterworth S, Beach C, Rigout M, Novoselov K S, Casson A J and Yeates S G 2017 All inkjet-printed graphenebased conductive patterns for wearable e-textile applications Journal of Materials Chemistry $C \mathbf{5}$ 11640-8

[25] Paul G, Torah R, Beeby S and Tudor J 2017 A printed, dry electrode Frank configuration vest for ambulatory vectorcardiographic monitoring Smart Materials and Structures 26025029

[26] Sinha S K, Noh Y, Reljin N, Treich G M, HajebMohammadalipour S, Guo Y, Chon K H and Sotzing G A 2017 Screen-Printed PEDOT:PSS Electrodes on Commercial Finished Textiles for Electrocardiography ACS Appl Mater Interfaces 9 37524-8

[27] Pani D, Dessi A, Saenz-Cogollo J F, Barabino G, Fraboni B and Bonfiglio A 2016 Fully Textile, PEDOT:PSS Based Electrodes for Wearable ECG Monitoring Systems IEEE Trans Biomed Eng 63 540-9

[28] Trindade I, Spranger P, Martins F, Miguel R and Santos Silva M 2014 Fully integrated embroidery process for smart textiles vol 3

[29] Trindade I G, Machado da Silva J, Miguel R, Pereira M, Lucas J, Oliveira L, Valentim B, Barreto J and Santos Silva M 2016 Design and Evaluation of Novel Textile Wearable Systems 
for the Surveillance of Vital Signals Sensors (Basel) 16

[30] Cho G, Jeong K, Paik M J, Kwun Y and Sung M 2011 Performance Evaluation of Textile-Based Electrodes and Motion Sensors for Smart Clothing IEEE Sensors Journal 11 3183-93

[31] Guo X, Huang Y, Cai X, Liu C and Liu P 2016 Capacitive wearable tactile sensor based on smart textile substrate with carbon black /silicone rubber composite dielectric Measurement Science and Technology 27045105

[32] Kalra A, Lowe A and Al-Jumaily A 2018 Critical review of electrocardiography measurement systems and technology Measurement Science and Technology 30012001

[33] Estepp J R and Christensen J C 2015 Electrode replacement does not affect classification accuracy in dual-session use of a passive braincomputer interface for assessing cognitive workload Front Neurosci 954

[34] Liu J, Ying D and Rymer W Z 2015 EMG burst presence probability: a joint time-frequency representation of muscle activity and its application to onset detection J Biomech 48 11937

[35] Logothetis I, Fernandez-Garcia R, Troynikov O, Dabnichki P, Pirogova E and Gil I 2019 Embroidered electrodes for bioelectrical impedance analysis: impact of surface area and stitch parameters Measurement Science and Technology

[36] Yao S and Zhu Y 2016 Nanomaterial-Enabled Dry Electrodes for Electrophysiological Sensing: A Review JOM 68 1145-55

[37] Taji B, Chan A D C and Shirmohammadi S 2018 Effect of Pressure on Skin-Electrode Impedance in Wearable Biomedical Measurement Devices IEEE Transactions on Instrumentation and Measurement 67 1900-12

[38] Li G, Wang S and Duan Y Y 2017 Towards gelfree electrodes: A systematic study of electrodeskin impedance Sensors and Actuators B: Chemical 241 1244-55

[39] Li G, Wang S and Duan Y Y 2018 Towards conductive-gel-free electrodes: Understanding the wet electrode, semi-dry electrode and dry electrode-skin interface impedance using electrochemical impedance spectroscopy fitting Sensors and Actuators B: Chemical 277 250-60

[40] Lee J W and Yun K S 2017 ECG Monitoring Garment Using Conductive Carbon Paste for Reduced Motion Artifacts Polymers (Basel) 9

[41] Cömert A, Honkala M and Hyttinen J 2013 Effect of pressure and padding on motion artifact of textile electrodes BioMedical Engineering OnLine 1226

[42] Cömert A and Hyttinen J 2015 Investigating the possible effect of electrode support structure on motion artifact in wearable bioelectric signal monitoring BioMedical Engineering OnLine 14 44

[43] Takeshita T, Yoshida M, Takei Y, Ouchi A, Hinoki A, Uchida H and Kobayashi T 2019 Relationship between Contact Pressure and Motion Artifacts in ECG Measurement with Electrostatic Flocked Electrodes Fabricated on Textile Scientific Reports 95897

[44] Shinwari M W, Zhitomirsky D, Deen I A, Selvaganapathy P R, Deen M J and Landheer D 2010 Microfabricated reference electrodes and their biosensing applications Sensors (Basel) 10 1679-715

[45] Degen T and Jaeckel H 2008 Continuous Monitoring of Electrode--Skin Impedance Mismatch During Bioelectric Recordings IEEE transactions on bio-medical engineering 55 17115

[46] Bierman W 1936 THE TEMPERATURE OF THE SKIN SURFACE JAMA 106 1158-62

[47] Evans S S, Repasky E A and Fisher D T 2015 Fever and the thermal regulation of immunity: the immune system feels the heat Nat Rev Immunol $15335-49$

[48] Logothetis I, Gkoutzeli D, Kagkas D, Vassiliadis S, Siores E and Pirogova E 2019 Thermoelectric Heat Patch for Clinical and Self-Management: Melanoma Excision Wound Care Ann Biomed Eng 47 537-48

[49] Bach A J, Stewart I B, Disher A E and Costello J T 2015 A comparison between conductive and infrared devices for measuring mean skin temperature at rest, during exercise in the heat, and recovery PLoS One $\mathbf{1 0} \mathrm{e} 0117907$

[50] Choi J K, Miki K, Sagawa S and Shiraki K 1997 Evaluation of mean skin temperature formulas by infrared thermography Int J Biometeorol 41 68-75

[51] Tyler C J 2011 The effect of skin thermistor fixation method on weighted mean skin temperature Physiol Meas 32 1541-7

[52] Mole R H 1948 The relative humidity of the skin J Physiol 107 399-411

[53] Gidik H, Bedek G and Dupont D 2016 Smart Textiles and their Applications, ed V Koncar (Oxford: Woodhead Publishing) pp 423-53

[54] Moradi B, Fernández-García R and Gil I 2020 Wearable high-performance meander ring dipole antenna for electronic-textile applications The Journal of The Textile Institute 111 178-82

[55] Gil I, Fernández-García R and Tornero J A 2018 Embroidery manufacturing techniques for textile dipole antenna applied to wireless body area network Textile Research Journal 89 1573-81

[56] Martinez-Estrada M, Moradi B, Fernández-Garcia $\mathrm{R}$ and Gil I 2018 Impact of Manufacturing Variability and Washing on Embroidery Textile Sensors Sensors 18 
[57] Dinh H 2013 Tales from the Development Frontier: The World Bank

[58] Murphy E J and Walker A C 1927 Electrical Conduction in Textiles. I The Dependence of the Resistivity of Cotton, Silk and Wool on Relative Humidity and Moisture Content The Journal of Physical Chemistry 32 1761-86

[59] Hu J Y, Li Y I and Yeung K W 2006 Clothing Biosensory Engineering, ed $\mathrm{Y} \mathrm{Li}$ and $\mathrm{A} \mathrm{S} \mathrm{W}$ Wong: Woodhead Publishing) pp 252-60

[60] Schindler W D and Hauser P J 2004 Chemical Finishing of Textiles, ed W D Schindler and P J Hauser: Woodhead Publishing) pp 1-6

[61] Logothetis I, Gil I, Dabnichki P and Pirogova E 2019 E textiles for bioelectrical impedance analysis (BIA). In: ABEC Conference 2019, Melbourne, Australia

[62] Shahzad A, Rasheed A, Khaliq Z, Qadir M B, Khan M Q, Hamdani S T A, Ali Z, Afzal A, Irfan M, Shafiq M and Kim I S 2019 Processing of metallic fiber hybrid spun yarns for better electrical conductivity Materials and Manufacturing Processes 34 1008-15

[63] Ageev I M, Rybin Y M and Shishkin G G 2016 Slow variations of the electroconductivity of distilled water Moscow University Physics Bulletin 71 556-61

[64] Golnabi H, R Matloob M, Bahar M and Sharifian M 2009 Investigation of electrical conductivity of different water liquids and electrolyte solutions vol 3 